\title{
The patchy growth mode: Modulation of the Au-Au interface via phenynyl ligands
}

\author{
Jianpeng Zong ${ }^{1}$, Quan Ren ${ }^{1}$, Xiaoli Tian ${ }^{1}$, Ting Xiang ${ }^{1}$, Yuhua Feng ${ }^{*}$ and Hongyu Chen ${ }^{1,2,3^{*}}$
}

\begin{abstract}
Surface ligands play critical roles in nanosynthesis and thus it is of great importance in expanding the scope of suitable ligands. In this work, we explore phenynyl ligands in modulating the Au-Au interface when growing Au domains on Au seeds. A patchy growth mode is observed where the emerging islands are flat-laying with holes and branches. This growth mode is distinctively different from the conventional facet-controlled growth using weak ligands, and the non-wetting island growth using strong ligands. Through manipulating the molecular structure and the packing of the phenynyl ligands on the Au seeds, the overgrown Au domains are continuously tuned, from patches to islands, extending the plasmon absorption peak into the near-infrared spectral range. We believe that the new ligand with intermediate affinity and the unusual growth mode would expand the control in both synthesis and application.
\end{abstract}

Keywords: phenynyls, binding affinity, Au patch, ligand packing, Au-Au hybrids

\section{INTRODUCTION}

Surface ligands play a pivotal role in nanochemistry and beyond. With electric or steric interactions, ligands stabilize colloidal nanoparticles and prevent their aggregation during collisions [1-3]. They are also essential in endowing nanoparticles with appropriate functional groups, for biological compatibility $[4,5]$, catalytic activity [6-9], chirality [10-13], and enhanced physical properties [14-16].

Ligands are widely used in nanosynthesis, as a means of synthetic control [17-21]. Indeed, most of the known methods for controlling the shape, size, and specific facets of nanoparticles are via the choice of ligands [22-26]. Despite the extensive studies, there are only few types of ligands known and they are either too weak or too strong.

The common ligands such as citrate ion, halide ions, oleylamine, cetyltrimethylammonium bromide (CTAB), and polyvinyl pyrrolidone (PVP) are all weak ligands. They are used with high concentrations, so that they would dynamically associate and dissociate on the nanoparticle surface [22]. In a typical synthesis, such a reversible ligand layer only slows down the rate of surface growth, depending on the ligand's on-off ratio [27]. The equivalent facets would grow equivalently, leading to highly symmetrical nanostructures.

In stark contrast, a strong thiol-ended ligand on $\mathrm{Au}$ surface could easily inhibit its growth at a very low concentration, ushering in rich growth modes [28]. For example, with all exposed Au surface inhibited by ligands, the selective deposition at the $\mathrm{Au}$-substrate interface could give a forest of ultrathin $\mathrm{Au}$ nanowires $[29,30]$. For nanoparticles suspended in a colloidal solution, dynamic control of the active growth sites makes it possible to sequentially modify the emerging nanostructure and achieve morphological control of wires $[31,32]$, plates, and caps [33].

In our recent study [34], a class of ligands with intermediate affinity to $\mathrm{Au}$, namely the phenynyl ligands, is discovered. Through in situ monitoring of ligand exchange by surfaceenhanced Raman scattering (SERS), the phenynyl ligands are found to be stronger than PVP and weaker than thiol-based ligands. Considering the lack of ligand types, they could offer a new window of synthetic exploration.

In this work, a new patchy growth mode of $\mathrm{Au}$ on $\mathrm{Au}$ seeds was achieved by modulating the Au seed NPs with phenynyl ligands. Through increasing the packing density of phenynyl ligands on the $\mathrm{Au}$ seed surface, the patchy structures with decreasing wetting degree were well controlled (Fig. 1a). In contrast to the strong thiol ligand which can be embedded by the deposit Au layer, the weaker phenynyl ligands lying on the surface of Au seeds showed lower resistance against the Au atom deposition. As a result, the growth of Au islands occurred in the hole of ligand layer can push away the ligands around, leading to the formation of $\mathrm{Au}$ islands in obvious wetting mode-the $\mathrm{Au}$ patches. In addition, unlike the slow reduction rate resulting from the formation of $\mathrm{Au}^{3+}$-thiol complexes, the fast reduction of $\mathrm{AuCl}_{4}{ }^{-}$gave rise to the multi-site growth of $\mathrm{Au}$ patches as the non-existence of $\mathrm{Au}^{3+}$-phenynyl complex. The exploration of the new phenynyl ligands led to the expansion of the range of interfacial energy modulation, and enriched the tool box for sophisticated nanosynthesis.

\section{EXPERIMENTAL SECTION}

\section{Materials and instruments}

All chemical reagents were purchased and used without further purification. Hydrogen tetrachloroaurate (III) trihydrate $\left(\mathrm{HAuCl}_{4}, 99.9 \%\right.$, Au $49 \%$ on metals basis, Alfa Aesar); tetra-

\footnotetext{
${ }^{1}$ Institute of Advanced Synthesis (IAS), School of Chemistry and Molecular Engineering, Jiangsu National Synergetic Innovation Centre for Advanced Materials, Nanjing Tech University, Nanjing 211816, China

${ }^{2}$ School of Science, Westlake University, Hangzhou 310023, China

${ }^{3}$ Institute of Natural Sciences, Westlake Institute for Advanced Study, Hangzhou 310024, China

* Corresponding authors (emails: ias_yhfeng@njtech.edu.cn (Feng Y); iashychen@njtech.edu.cn (Chen H))
} 


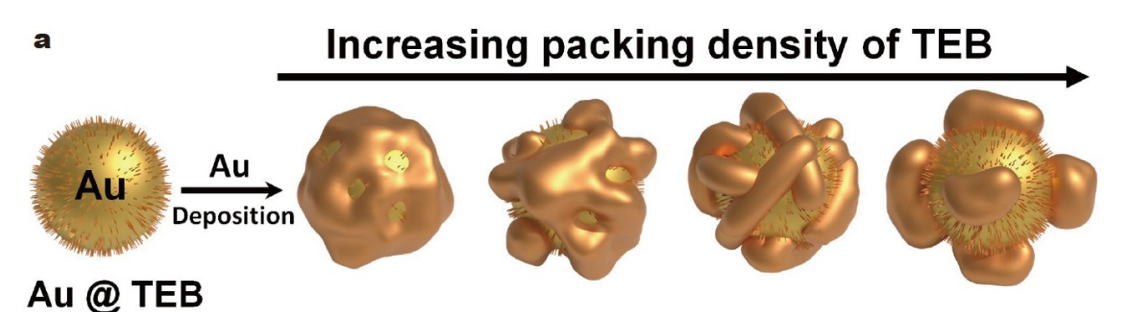

\section{Au @ TEB}
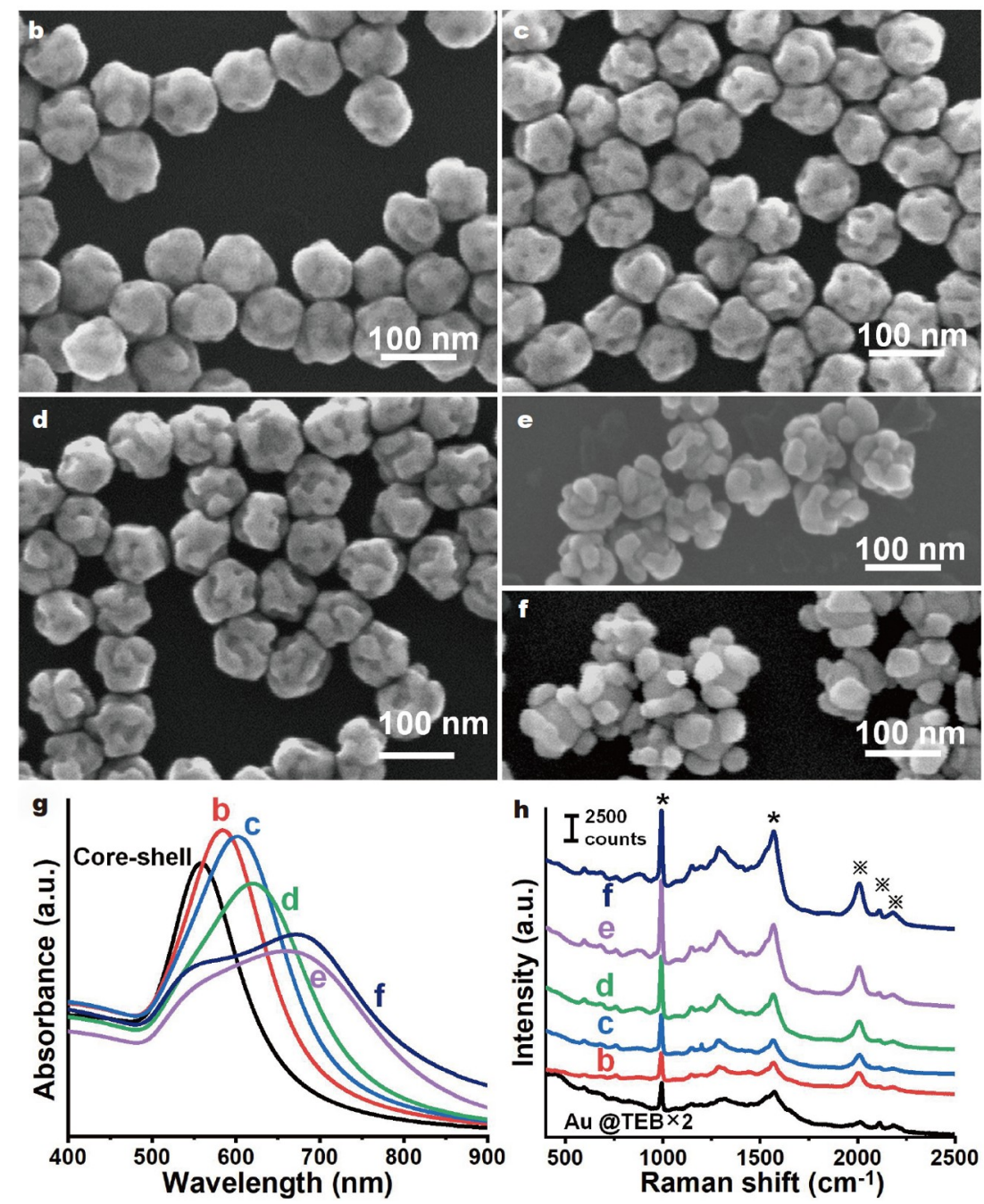

Figure 1 (a) Schematics of the synthesis of Au patchy structures by varying the amount of TEB ligands. SEM images of the Au patchy structures synthesized with TEB ligands of (b) 13, (c) 25, (d) 50, (e) 100, and (f) $200 \mu \mathrm{mol} \mathrm{L}^{-1}$. (g, h) The absorption and SERS spectra of the Au patchy structures.

decyltrimethylammonium bromide (TTAB) was purchased from Tokyo Chemical Industry Co., Ltd. (TCI); $\mathrm{NaOH}$ and $\mathrm{N}, \mathrm{N}$ dimethylformamide (DMF) were purchased from Sinopharm Chemical Reagent Co. Ltd.; $L$-ascorbic acid was purchased from Sigma Aldrich. 1,3,5-Triethynylbenzene (TEB), 1,3-diethynylbenzene (1,3-DEB), 1,4-diethynylbenzene (1,4-DEB), and phenylacetylene (PhA) were purchased from Aladdin; 1,2-DEB was purchased from Zhengzhou RUKE Biotech Co., Ltd.; 1,3,5-tri (prop-1-yn-1-yl)benzene was purchased from Shanghai TENSUS Biotech CO. Ltd.; deionized water (resistance $>18.2 \mathrm{M} \Omega \mathrm{cm}^{-1}$ ) was used in all reactions.

Scanning electron microscopy (SEM; FEI, Quanta 250 FEG) was used to characterize the morphology of $\mathrm{Au}$ structures at $30 \mathrm{kV}$. Absorption spectra were obtained by diluting the product five times and collected on a Lambda $750 \mathrm{UV}-\mathrm{Vis}$ spectrophotometer. SERS was collected by measuring the as-synthesized sample solutions on a portable Raman analyzer (Accuman SR510Pro) equipped with the excitation wavelength of $785 \mathrm{~nm}$. The laser power was $350 \mathrm{~mW}$ and the integration times were $10 \mathrm{~s}$ for all samples.

Synthesis of $60 \mathrm{~nm}$ citrate-stabilized Au NPs and ligand exchange between citrate and TTAB

The Au NPs were synthesized based on the citrate reduction and grown to $60 \mathrm{~nm}$ by a multi-step seed-mediated growth method [28]. Specifically, $0.375 \mathrm{~mL}$ of sodium citrate solution (1 wt\%) was added to $25 \mathrm{~mL}$ of boiling $\mathrm{HAuCl}_{4}$ aqueous solution $\left(0.1 \mathrm{mg} \mathrm{mL}^{-1}\right)$. After $10 \mathrm{~min}$, the solution colour changed to red 
indicating that $40 \mathrm{~nm} \mathrm{Au} \mathrm{NPs} \mathrm{were} \mathrm{synthesized} \mathrm{and} \mathrm{then} \mathrm{con-}$ tinuously grown by the seed growth method. In the first round, after being refluxed for another $25 \mathrm{~min}, 25 \mathrm{~mL}$ of boiled water was added into the red $\mathrm{Au}$ NPs solution, followed by the addition of $50 \mu \mathrm{L}$ of $\mathrm{NaOH}$ solution $\left(6.6 \mathrm{mg} \mathrm{mL}^{-1}\right), 0.25 \mathrm{~mL}$ of sodium citrate solution ( $1 \mathrm{wt} \%$ ) and $0.25 \mathrm{~mL}$ of $\mathrm{HAuCl}_{4}$ solution $\left(10 \mathrm{mg} \mathrm{mL}^{-1}\right)$. The solution continued to boil for $30 \mathrm{~min}$ to ensure the complete reduction of $\mathrm{HAuCl}_{4}$. In the second round, $50 \mathrm{~mL}$ of boiled water, $100 \mu \mathrm{L}$ of $\mathrm{NaOH}$ solution, $0.5 \mathrm{~mL}$ of sodium citrate solution ( $1 \mathrm{wt} \%$ ) and $0.5 \mathrm{~mL}$ of $\mathrm{HAuCl}_{4}$ solution $\left(10 \mathrm{mg} \mathrm{mL}^{-1}\right)$ were added in sequence in the same way and repeated three times. In the final round, $100 \mu \mathrm{L}$ of $\mathrm{NaOH}$ solution, $0.5 \mathrm{~mL}$ of sodium citrate solution (1 wt\%), and $0.5 \mathrm{~mL}$ of $\mathrm{HAuCl}_{4}$ solution $\left(10 \mathrm{mg} \mathrm{mL}^{-1}\right)$ were added in sequence and repeated twice. Finally, the citrate-stabilized $60 \mathrm{~nm} \mathrm{Au} \mathrm{NPs} \mathrm{were}$ synthesized.

Pre-synthesized citrate-stabilized Au NPs $(40 \mathrm{~mL})$ were centrifuged at $4000 \mathrm{r} \mathrm{min}^{-1}$ for $15 \mathrm{~min}$ to remove the supernatant, and the sediment was redispersed in $20 \mathrm{~mL}$ of pure water. Meanwhile, $20 \mathrm{~mL}$ of TTAB solution $\left(20 \mathrm{mmol} \mathrm{L}^{-1}\right)$ was stirred vigorously under magnetic stirring $\left(1400 \mathrm{r} \mathrm{min}^{-1}\right)$. After being dissolved completely, $20 \mathrm{~mL}$ of $\mathrm{Au}$ NP solution was added dropwise to the TTAB solution under vigorous stirring. After the complete addition, the Au NPs/TTAB solution was continuously stirred for 2-4 h. Finally, $10 \mathrm{mmol} \mathrm{L}^{-1} \mathrm{TTAB}$-stabilized Au NP solution was obtained and stored at $30^{\circ} \mathrm{C}$ to prevent the precipitation of TTAB.

\section{Synthesis of Au-Au patchy and island nanostructures}

DMF solution of different concentrations of TEB was added to $1 \mathrm{~mL}$ of the above solution of TTAB-stabilized Au NPs under vortex conditions, and incubated at $60^{\circ} \mathrm{C}$ for $2 \mathrm{~h}$. After cooling to room temperature (RT), $50 \mu \mathrm{L}$ of $L$-ascorbic acid $\left(20 \mathrm{mmol} \mathrm{L}^{-1}\right)$ and $50 \mu \mathrm{L}$ of $\mathrm{HAuCl}_{4}\left(10 \mathrm{mmol} \mathrm{L}^{-1}\right)$ were quickly added to the above solution under vortex stirring. The mixed solution was placed at room temperature and reacted for $3 \mathrm{~h}$. After the reaction, the products were centrifuged at $4500 \mathrm{r} \mathrm{min}^{-1}$ for $10 \mathrm{~min}$ and washed with pure water to remove excess TTAB surfactant, which was convenient for SEM characterization. The experimental procedure to increase the reaction rate was implemented simply by adding different amounts of $\mathrm{NaOH}$ before adding $L$-ascorbic acid and $\mathrm{HAuCl}_{4}$. The products were diluted five times for ultraviolet-visible (UV-Vis) absorption spectra, and the reaction stock solution was directly used for SERS characterization.

Complexation of 5-amino-2-mercaptobenzimidazole (AMBI) and TEB with $\mathrm{HAuCl}_{4}$

Different concentrations of AMBI or TEB were added to $1 \mathrm{~mL}$ of TTAB $\left(10 \mathrm{mmol} \mathrm{L}^{-1}\right)$ solution under vortex, followed by the addition of $\mathrm{HAuCl}_{4}\left(10 \mathrm{mmol} \mathrm{L}^{-1}\right)$ solution. After reacting for $10 \mathrm{~min}$, the reaction solution was diluted five times for UV-Vis spectra test.

\section{RESULTS AND DISCUSSION}

For the compatibility of the Au seeds with phenynyl ligands, the citrate-stabilized Au NPs $(d=63 \mathrm{~nm})$ were transformed into $10 \mathrm{mmol} \mathrm{L}^{-1}$ TTAB solution by ligand exchange (Fig. S1). Then the TTAB Au seeds were incubated with TEB ligands at $60^{\circ} \mathrm{C}$ for $2 \mathrm{~h}$ (Fig. S2). After cooling to RT, reductant ascorbic acid (AA) and $\mathrm{HAuCl}_{4}$ precursor were added in sequence under vigorous vortex to initiate the growth of Au on Au@TEB seeds. The resulting solution was placed undisturbed at RT for $3 \mathrm{~h}$, during which the color changed from light red to purple and blue (Fig. S3). The product was separated by centrifugation at $4000 \mathrm{r} \mathrm{min}^{-1}$ for $10 \mathrm{~min}$. The concentrated NPs collected at the bottom of the centrifuge tube were used for SEM characterizations.

As shown in Fig. 1b, when the concentration of TEB ligands ([TEB]) in pre-incubation with Au seeds was $13 \mu \mathrm{mol} \mathrm{L}^{-1}$ (the final concentration), Au NPs with craters were obtained. Compared with Au seeds, the average size increase is $88 \mathrm{~nm}$, indicating the formation of a 12-nm Au layer on Au seeds. Unlike the formation of uniform $\mathrm{Au}$ layer on $\mathrm{Au}$ seeds in a typical seeded growth process, the newly grown Au layer is not smooth. We speculate that the presence of TEB ligands on $\mathrm{Au}$ seeds interfered with the uniform deposition of $\mathrm{Au}$, leaving craters on the surface. To verify the ligand effect of TEB, their concentrations in pre-incubation were varied in two directions.

As can be seen in Fig. S4, when the [TEB] was decreased to $7 \mu \mathrm{mol} \mathrm{L}{ }^{-1}$, the surface of the resultant Au NPs became relatively smoother, and the crater size was significantly decreased. While in the absence of TEB ligands, uniform Au NPs with smooth surface were obtained. This result revealed that the stronger coordination of TEB on Au led to a higher on-off ratio than that of TTAB, which induced the non-uniform growth of Au layer on the surface of Au@TEB NPs.

When the [TEB] was increased to 25 and $50 \mu \mathrm{mol} \mathrm{L}{ }^{-1}$, both the number and the size of the craters increased obviously (Fig. 1c, d). As shown in Fig. 1d, the round craters changed to channel-like gaps, leading to more exposure of the seed surface. From Fig. 1b-d, the linearly decreased wetting of $\mathrm{Au}$ on $\mathrm{Au}$ seeds is inversely proportional to the increase of [TEB]. As the increase of [TEB] in pre-incubation led to the formation of TEB layer with a higher density, we speculate that the change of the surface morphology should arise from the increased density of TEB on Au seeds. Specifically, the densely packed TEB ligands are more difficult to be pushed away during Au atom deposition. The denser the TEB ligands, the lower wetting degree of $\mathrm{Au}$ growth on Au@TEB seeds.

To confirm the above hypothesis, the [TEB] was further increased to $100 \mu \mathrm{mol} \mathrm{L}{ }^{-1}$. Interestingly, the growth of $\mathrm{Au}$ transformed from layer mode to island mode, yielding stripeshaped Au domains separately distributed on the Au seed surface with increased non-wetting degree (Fig. 1e). Here, to distinguish from the conventional spherical Au islands [35-37], the above-mentioned separated Au domains with large wettability were defined as Au patches [38].

At high [TEB] of $200 \mu \mathrm{mol} \mathrm{L}{ }^{-1}$, the interval between the Au patches increased significantly, meanwhile, the height of the patch was also increased obviously. Except for the wetting contact of the bottom on Au seeds, the Au patches are similar to the shape of conventional Au islands. When the [TEB] was higher than $200 \mu \mathrm{mol} \mathrm{L}^{-1}\left(300 \mu \mathrm{mol} \mathrm{L}^{-1}\right.$, Fig. S3), the same $\mathrm{Au}$ patchy structures with that in Fig. If were obtained, indicating the ligand density on seeds had reached the ceiling.

To check the effect of TTAB surfactant on the Au patchy structures, citrate-stabilized $\mathrm{Au}$ NPs were transferred into $10 \mathrm{mmol} \mathrm{L}^{-1}$ of $\mathrm{CTAB}$ and cetyltrimethylammonium chloride (CTAC) solutions. Under the same synthetic conditions with that in Fig. 1, similar Au patchy structures were obtained for CTAB-Au seeds (Fig. S5). Nevertheless, for CTAC-Au seeds, as 
the more positive reduction potential of the $\left[\mathrm{AuCl}_{4}\right]^{-}$complex [39], the reduction rate is much faster than that of $\left[\mathrm{AuBr}_{4}\right]^{-}$. The fast reduction led to higher $\mathrm{Au}^{0}$ oversaturation rate during the nucleation stage, causing the formation of multiple small $\mathrm{Au}$ islands on the surface of $\mathrm{Au}$ seeds at higher TEB concentrations (> $50 \mu \mathrm{mol} \mathrm{L}{ }^{-1}$, Fig. S6).

In addition to the [TEB], the pre-incubation conditions of $\mathrm{Au}$ seeds with TEB ligands were found to be keystep for the formation of Au patchy structures. When the pre-incubation of $\mathrm{Au}$ seeds with $100 \mu \mathrm{mol} \mathrm{L}^{-1}$ TEB was carried out at RT for $2 \mathrm{~h}, \mathrm{Au}$ NPs in $82 \mathrm{~nm}$ diameter with smooth surface were obtained (Fig. 2a). Without pre-incubation, Au NPs with similar diameters (Fig. S7) were produced. The results of these control experiments indicate the key role of pre-incubation at elevated temperatures. It is known that the incubation of ligands with $\mathrm{Au}$ seeds at high temperatures would accelerate the establishment of the dynamic equilibrium of ligand on their surfaces [40]. At equilibrium state, the densely ordered ligand packing provides additional stability during Au atom deposition, leading to the change of the growth mode of Au thereon. Similar to the typical seeded growth process, the loosely packed ligands without or after RT incubation would be pushed away during Au atom deposition, giving rise to the formation of uniform larger $\mathrm{Au}$ NPs.

To verify the above hypothesis, a control experiment was carried out by adding 1 equiv. of TTAB-stabilized Au NPs to a solution of $\mathrm{Au}$ NPs which had just undergone $60^{\circ} \mathrm{C}$ incubation with $100 \mu \mathrm{mol} \mathrm{L}^{-1}$ TEB. After Au deposition, the resulting product showed roughly 1:1 ratio of core-shell to patchy NPs (Fig. 2b and Fig. S8). The different growth modes in the same solution argue against other possible influencing factors [28].

In absorption spectra (Fig. 1g), compared with the Au NPs after seeded growth in absence of TEB ligand, the formation of $\mathrm{Au}$ NPs with craters on the surface led to the redshift of the $\mathrm{Au}$ absorption from $558 \mathrm{~nm}$ to 584,601 and $620 \mathrm{~nm}$. With the increase of the number of craters and the separation of $\mathrm{Au}$ patches, besides the $\mathrm{Au}$ absorption at $540 \mathrm{~nm}$, new peaks appeared at 661 and $671 \mathrm{~nm}$ for structures of Fig. 1d, e. Considering the roughly isotropic structure, we believe that the absorption at longer wavelengths should arise from the coupling of the $\mathrm{Au}$ patches [41-43].

To confirm the presence of TEB ligands in the resulting Au patchy structures, SERS measurement of the as-synthesized solution was carried out by using a 785-nm-wavelength laser. As shown in Fig. 1h, compared with the SERS spectrum of Au@TEB and Raman spectrum of TEB powder (Fig. S9), the characteristic Raman peaks were observed in all the samples. The SERS intensity increased with increasing amount of TEB ligands added in the pre-incubation step.

In SERS spectra, the Raman shifts at 991 and $1569 \mathrm{~cm}^{-1}$ can be assigned to the breathing and stretching vibration of the aromatic ring, and the 2006, 2109 and $2178 \mathrm{~cm}^{-1}$ peaks are characteristic stretching modes of the $-\mathrm{C} \equiv \mathrm{C}$ group [44], from which the binding of the TEB on Au surface can be confirmed.

From the density functional theory (DFT) calculation result [45], only when the terminal hydrogen was deprotonated, the upright $\mathrm{Au}-\mathrm{C} \sigma$ bond can form between the $-\mathrm{C} \equiv \mathrm{C} \cdot$ and $\mathrm{Au}$, which is stronger than that of the $\mathrm{Au}-\mathrm{S}$ coordination bond. Considering that the abstraction of the terminal hydrogen in $-\mathrm{C} \equiv \mathrm{CH}$ can only occur in the presence of strong base of $n$ butyllithium under strictly inert atmosphere or the catalyst, no $\mathrm{Au}-\mathrm{C} \sigma$ bond can form in our system [46,47]. The control experiment established that from the disappearance of the SERS of TEB, 11-mercaptoundecanoic acid at 1:1 ratio could completely replace the TEB ligand on $\mathrm{Au}$ seeds within $2 \mathrm{~h}$ under $60^{\circ} \mathrm{C}$ incubation (Fig. S10). Combined with that no stretching vibration peak belonging to the covalent $\mathrm{Au}-\mathrm{C}$ bond was found in the range of $400-420 \mathrm{~cm}^{-1}$ [48], instead the upright configuration, the TEB ligands should coordinate on the Au surface in a flat-lying mode through the formation of $\mathrm{d}-\mathrm{p} \pi$ bond via the $-\mathrm{C} \equiv \mathrm{C}-2 \mathrm{p}$ and $\mathrm{Au} 5 \mathrm{~d}$ orbitals $[34,49]$.

It should be noted that, when all of the terminal hydrogen in TEB was replaced by $-\mathrm{CH}_{3}$ group, no structural modulation effect was observed even at a high concentration of $0.8 \mathrm{mmol} \mathrm{L}^{-1}$ (Fig. S11). In addition, no $-\mathrm{C} \equiv \mathrm{C}-$ Raman shift was observed in SERS spectra. These results revealed that only terminal phenynyls can be used to tune the Au patchy structures. The possible reason is that the steric hindrance of the end group led to decreased strength of $\mathrm{d}-\mathrm{p} \pi$ bond between $-\mathrm{C} \equiv \mathrm{C}-$ and $\mathrm{Au}$.

On the basis of the above study, TEB analogues 1,3-DEB and phenylacetylene (PhA) were employed to study the structural tuning effect from various numbers of alkyne groups. As shown in Fig. 3a, the Au patchy structure was synthesized at $0.8 \mathrm{mmol} \mathrm{L}^{-1}$ of 1,3 -DEB. Under the same conditions, the concentration of 1,3-DEB ligands required to effectively tune the $\mathrm{Au}$ patchy structure was higher than that of TEB (Figs S12 and S13), only $1 / 8$ of the 1,3-DEB concentration. While for $\mathrm{PhA}, 80 \mathrm{~nm}$ $\mathrm{Au}$ NPs with smooth surface were obtained at the same concentration of $0.8 \mathrm{mmol} \mathrm{L} \mathrm{L}^{-1}$, indicating no structural tuning effect (Fig. 3b).
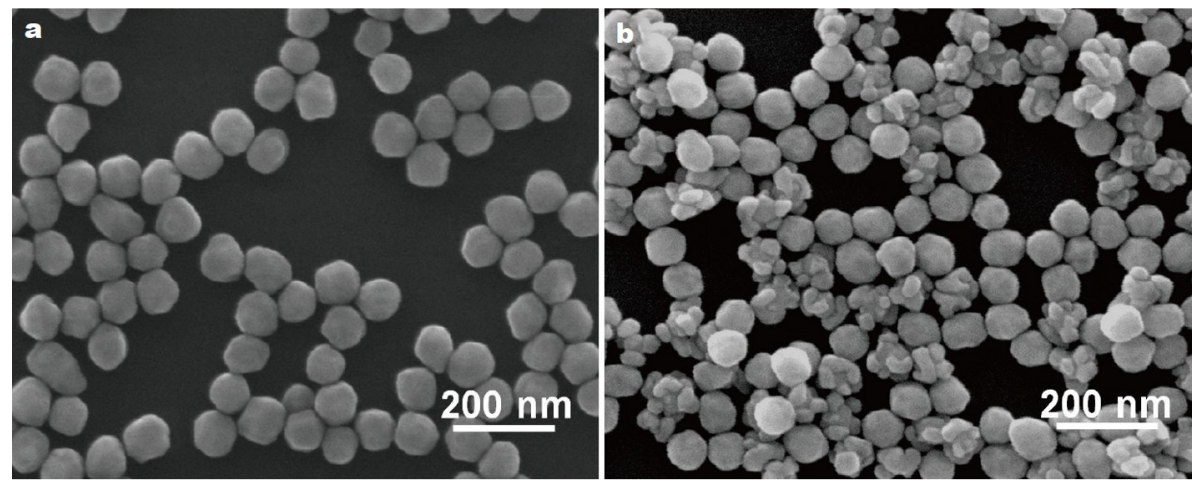

Figure 2 SEM images of the Au structures obtained by the Au seeds. (a) RT incubation of Au seeds with 0.1 mmol L ${ }^{-1}$ TEB; (b) a mixture of the bare Au NPs and $\mathrm{Au}$ seeds pre-incubated with $0.1 \mathrm{mmol} \mathrm{L}^{-1} \mathrm{TEB}$ at $60^{\circ} \mathrm{C}$ in $1: 1$ ratio. 

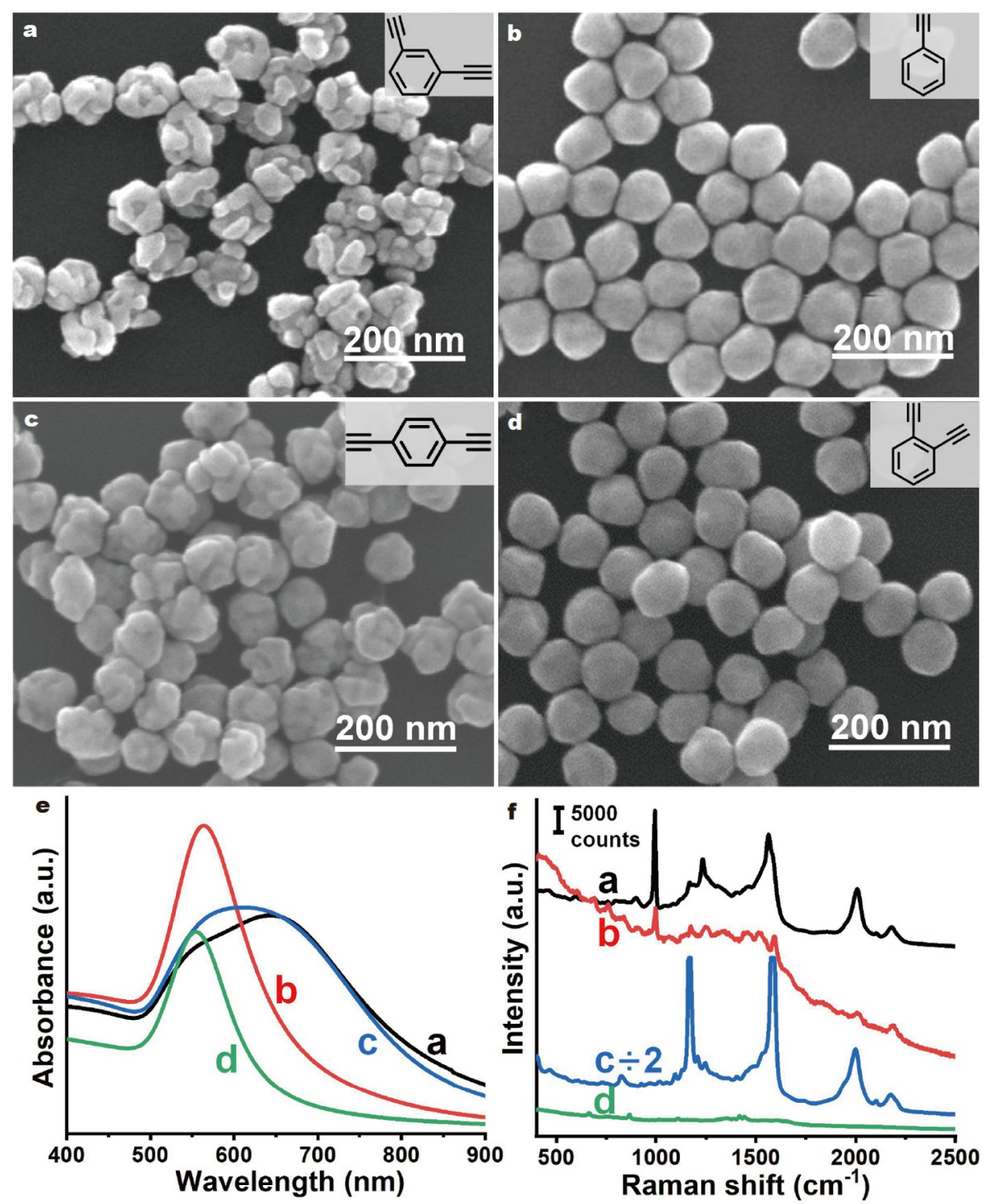

Figure 3 SEM images of the Au NPs synthesized using different phenynyl ligands. (a) 1,3-DEB, (b) PhA, (c) 1,4-DEB, (d) 1,2-DEB; (e, f) the absorption and SERS spectra of the Au NPs in (a-d).

In addition, for DEB ligands, the relative position of the two alkynyl groups can also affect the abilities in tuning the patchy structures. Under the same concentration of $0.8 \mathrm{mmol} \mathrm{L}^{-1}, 1,4$ DEB led to the formation of Au patchy structures (Fig. 3c) similar to that in Fig. 1b, indicating its weak structural tuning ability (Fig. S14). While for 1,2-DEB ligands at $0.8 \mathrm{mmol} \mathrm{L}^{-1}$, no patchy structure was observed (Fig. 3d).

As shown in Fig. 3e, the absorptions of the Au NPs and patchy structures were similar to that in Fig. 1g. Specifically, the redshift was due to the increase in size, and the appearance of the new absorption peak arised from the coupling of Au patches. In SERS spectra, the characteristic Raman shift of the different ligands were all observed, confirming the presence of the ligands on the resulting structures (Fig. 3f and Fig. S15).

Based on the above results, for the phenynyl ligands, their ability of structural tuning decreased in the sequence of TEB, 1,3-DEB, 1,4-DEB, PhA, and 1,2-DEB. We speculate that the difference results from the different strengths of the phenynyl$\mathrm{Au}$ affinity. The ligand exchange of alkynyl ligands evidenced that the order of the coordination strength with $\mathrm{Au}$ was almost the same as their structural tuning abilities (Tables S1 and S2).

There are several critical differences between thiol and phenynyl ligands in structural control. The S-Au bond is known to be very strong, especially after deprotonation [50]. The binding of thiol ligands via single-atom anchoring point makes them easily trapped in defect sites, even after repeated washing [51]. Thus, the combination of the two factors explains why it is difficult to push away thiol ligands, even at low concentrations. It can even cause them to be embedded between the seed and the overcoating metal layer $[52,53]$. As a result, non-wetting growth of the $\mathrm{Au}$ island was obtained due to the preferential $\mathrm{Au}$ deposition on the fresh Au surface, where ligand close packing is not yet established (Fig. 4a).

In contrast, the phenynyl ligands are weaker and bind through the two-carbon "flat-lying" mode along with the bulky phenyl group, making it difficult to be trapped in defects (Fig. 4b). The weak affinity also leads to a lower partition coefficent (surface ligand density/ligand concentration) and more rapid ligand dynamics. That is perhaps why the phenynyl ligands are easier to be pushed away at low ligand density, and why Au domain is 


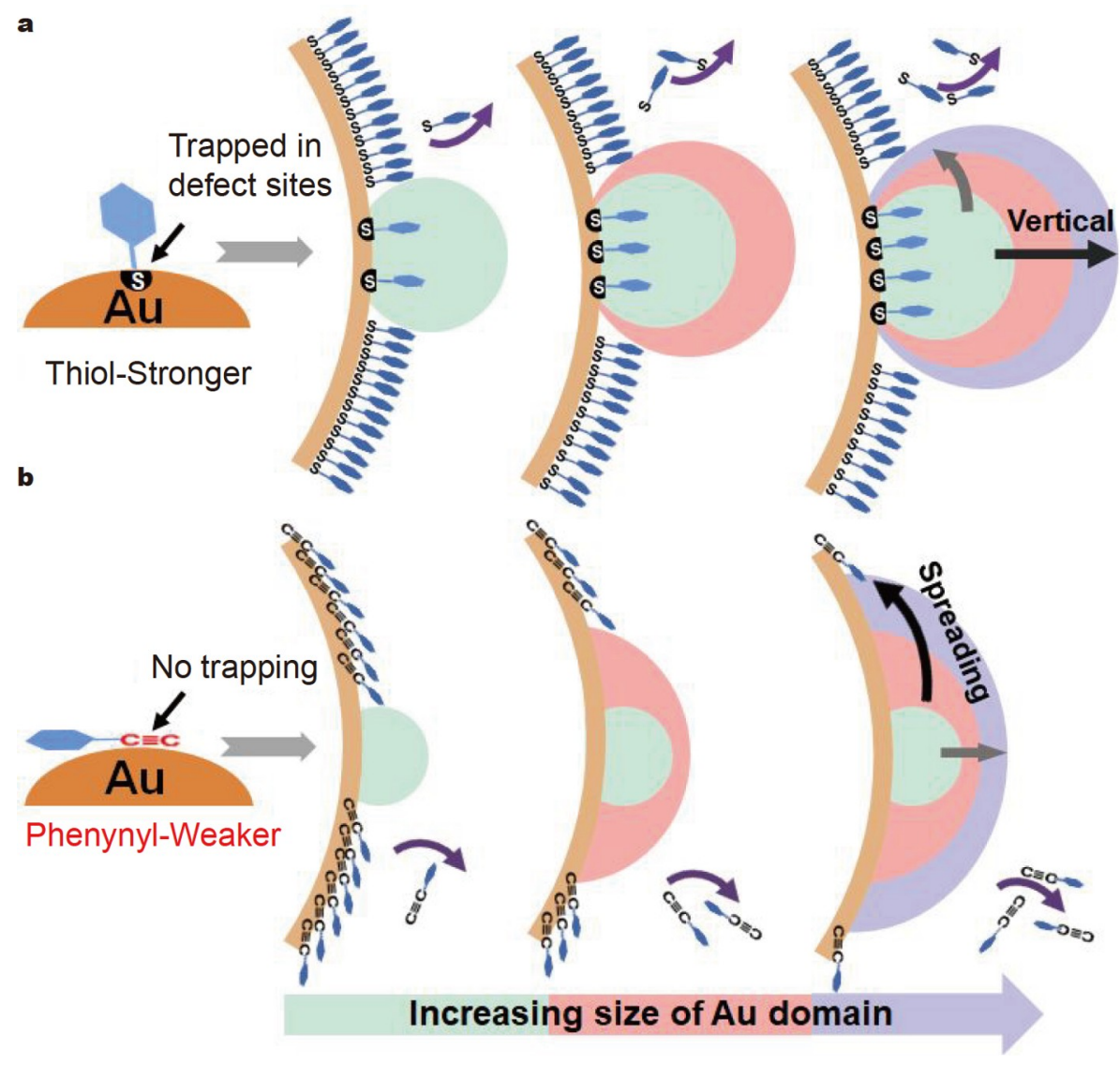

Figure 4 Schematics illustrating the difference in the growth of Au domains on Au seeds modified with (a) thiol, and (b) phenynyl ligands.

prone to spread on the seed surface with higher wetting degree.

The stripes of $\mathrm{Au}$ domains are most revealing: as the preferential deposition on the fresh $\mathrm{Au}$ surface continues, the $\mathrm{Au}$ domain has to push away the nearby old surface of the seed, in order to maintain a favorable wetting configuration (Fig. 4b). But such spreading is obviously anisotropic. A reasonable explanation is that the stripes represent the initial gaps between the ligand patches, because the peripheral ligands could be more easily removed.

With the increase of phenynyl ligand concentration, the stripes became shorter, and only at the $200 \mu \mathrm{mol} \mathrm{L}^{-1}$ concentration, did the Au domains turned into non-wetting islands of near-spherical shape. It appears that the denser ligands lead to a change of the binding affinity, at least at the gaps of ligand patches. In the above discussion, the ligand concentration (Fig. 1b-f), the process of ligand incubation (Fig. 2a, b) and the number and position of the ethynyl groups (Fig. 3a-d) are all critical factors, highlighting the importance of ligand packing.

It should be noted that compared with the thiol ligands, the colour changes in phenynyl ligand-controlled $\mathrm{Au}$ patchy structures were much faster. In contrast to the thiol-induced single island growth, multi-patch growth is always obtained for TEB ligand. This should result from the fast growth kinetics. On the one hand, the weak affinity of the phenynyls on Au made them to be easily pushed away. More importantly, the $\mathrm{HAuCl}_{4}$ precursor was reduced to $\mathrm{Au}$ atoms in a much faster rate in the phenynyl system than that in the presence of thiol ligand. The reason is that thiols can form stable complex with $\mathrm{Au}^{3+}$, which leads to the significant decrease of Au reduction rate. However, the phenynyl ligands cannot form stable complex with $\mathrm{Au}^{3+}$, and thus will not affect their reduction rate. According to the Depletion Sphere model [54], the higher Au atom oversaturation would lead to smaller depletion sphere on seed surface, thus induce multi-site nucleation and growth.

To conform the above hypothesis, TEB and AMBI were selected to study their complexing effects with $\mathrm{HAuCl}_{4}$. In a typical procedure, the ligand and $\mathrm{HAuCl}_{4}$ were sequentially added into $10 \mathrm{mmol} \mathrm{L}^{-1} \mathrm{TTAB}$ solution and incubated at RT for 10 min before the absorption spectrum collection. For AMBI ligand, when the ratio of $\mathrm{AMBI}: \mathrm{HAuCl}_{4}$ increased from 0 to 3.2: 1 , the final colours ranged from light yellow to grey. From absorption spectra (Fig. 5a), with the increase of AMBI, the peak at $390 \mathrm{~nm}$ derived from the TTAB:Au ${ }^{3+}$ complex decreased gradually. After the peak at $390 \mathrm{~nm}$ disappeared, a new peak at $310 \mathrm{~nm}$ can be observed which can be assigned as the absorption of AMBI: $\mathrm{Au}^{3+}$ complex [55]. The intensity increased linearly with the increase of AMBI content, indicating the formation of increased amount of AMBI:Au ${ }^{3+}$ complexes. While under the same conditions, the absorption of TTAB: $\mathrm{Au}^{3+}$ complex at $390 \mathrm{~nm}$ showed no change with the increasing amount of phenynyl ligands (Fig. 5b). At the same time, no new absorption was observed. Based on the above results, TEB ligands cannot form stable complex under above synthetic conditions.

To verify the effect of the growth kinetics on the Au patchy structures, different amounts of $\mathrm{NaOH}$ were introduced to accelerate the reduction of $\mathrm{HAuCl}_{4}$, while all other conditions were remained unchanged (Figs S16 and S17). As shown in Fig. $6 a, b$, when the $[\mathrm{NaOH}]$ was increased from 1.3 to 

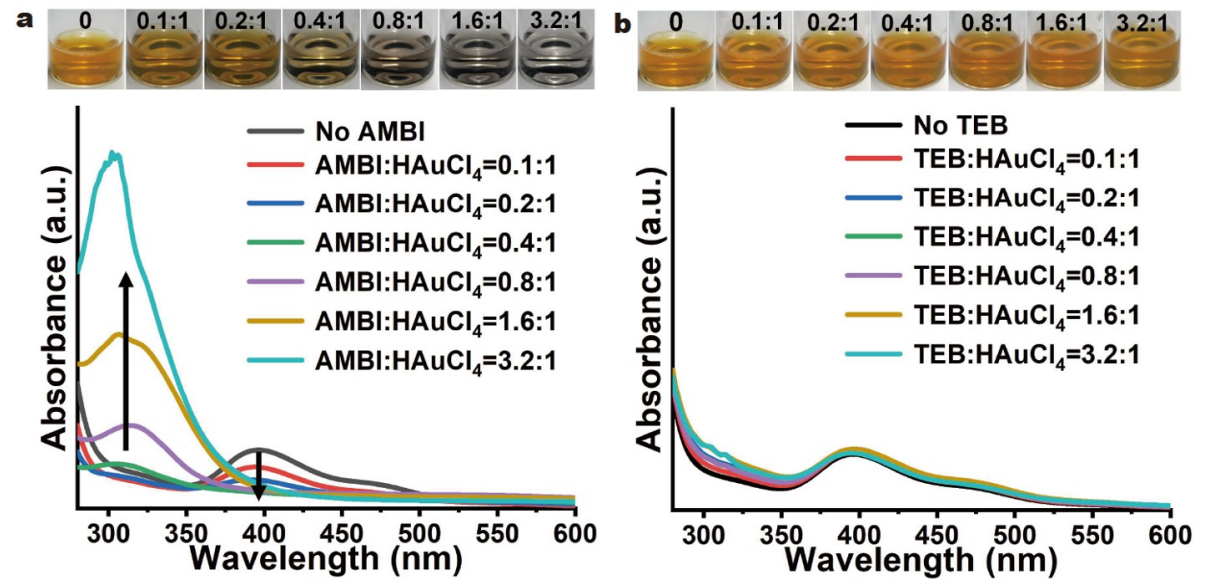

Figure 5 The photographs and absorption spectra of the solutions of $\mathrm{HAuCl}_{4}$ mixed with (a) AMBI, and (b) TEB in different ratios.
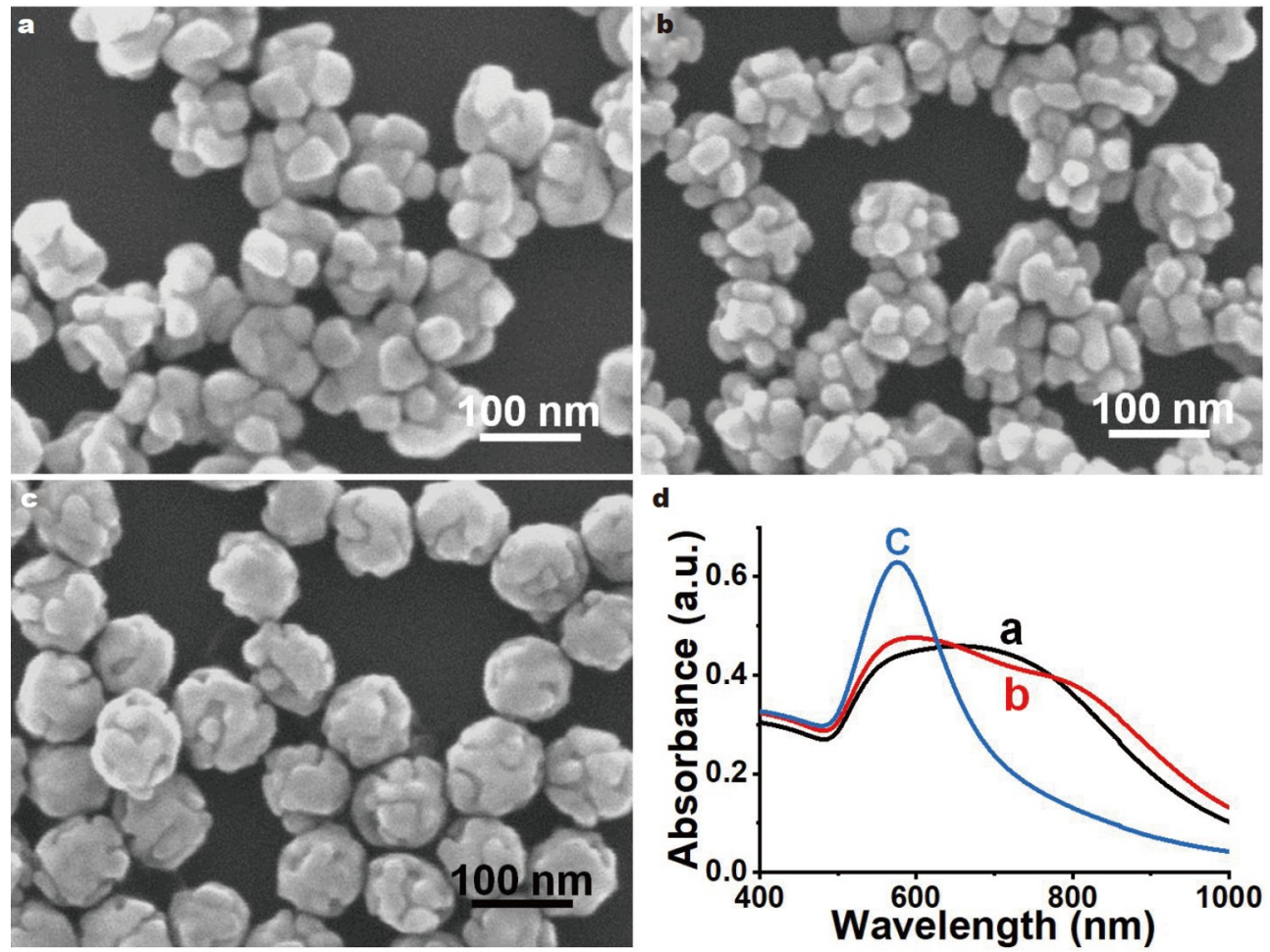

Figure 6 SEM images of the Au patchy structures synthesized with $100 \mu \mathrm{mol} \mathrm{L} \mathrm{L}^{-1}$ TEB under different concentrations of $\mathrm{NaOH}$ : (a) 1.3, (b) 1.8, and (c) $2.5 \mathrm{mmol} \mathrm{L}^{-1}$. (d) The absorption spectra of the Au patchy structures in (a-c).

$1.8 \mathrm{mmol} \mathrm{L}^{-1}$, the number of the Au patches increased obviously, accompanied with the decrease in size. When [NaOH] was increased to $2.5 \mathrm{mmol} \mathrm{L}^{-1}$, most of the Au seeds were covered with $\mathrm{Au}$ patches (Fig. 6c). Similar to the crown shyness phenomena, channel-like gaps between the neighbouring patches formed possibly due to the compact packing of TEB ligands thereon. As shown in Fig. 6d, in addition to the absorption at $590 \mathrm{~nm}$, a new peak appeared at 700 and $800 \mathrm{~nm}$ for Fig. 6a, b, which should arise from the coupling of the $\mathrm{Au}$ patches.

\section{CONCLUSIONS}

In summary, through interfacial modulation by the new type of phenynyl ligands, the growth of Au patches with different wetting degrees on the Au seed NPs was successfully achieved. The formation of the Au patchy structures is due to the moderate affinity of phenynyl ligands on Au. By varying the ligand density and packing state on Au seeds, the Au patchy structures can be continuously tuned in the number, the size and the wetting degrees of the patches. As a result of structural variabilities, the localized surface plasmon resonance (LSPR) of the Au patchy structures can be readily tuned within the Vis-near-infrared (NIR) spectral range.

The exploitation of new types of surface ligands with moderate affinity led to new wetting growth of Au patches on $\mathrm{Au}$ seeds. Beyond the enrichment of the ligand scope, we believe that the 
new control may assist the development of new sophisticated nanostructures and promote their applications.

\section{Received 5 November 2021; accepted 21 December 2021; published online 15 February 2022}

1 Boles MA, Ling D, Hyeon T, et al. The surface science of nanocrystals. Nat Mater, 2016, 15: 141-153

2 Zhu H, Prince E, Narayanan P, et al. Colloidal stability of nanoparticles stabilized with mixed ligands in solvents with varying polarity. Chem Commun, 2020, 56: 8131-8134

3 Kang H, Buchman JT, Rodriguez RS, et al. Stabilization of silver and gold nanoparticles: preservation and improvement of plasmonic functionalities. Chem Rev, 2019, 119: 664-699

4 Zhan N, Palui G, Safi M, et al. Multidentate zwitterionic ligands provide compact and highly biocompatible quantum dots. J Am Chem Soc, 2013, 135: 13786-13795

$5 \mathrm{Na} \mathrm{HB}$, Palui G, Rosenberg JT, et al. Multidentate catechol-based polyethylene glycol oligomers provide enhanced stability and biocompatibility to iron oxide nanoparticles. ACS Nano, 2012, 6: 389-399

6 Xue T, Lin Z, Chiu CY, et al. Molecular ligand modulation of palladium nanocatalysts for highly efficient and robust heterogeneous oxidation of cyclohexenone to phenol. Sci Adv, 2017, 3: e1600615

7 Ogiwara $\mathrm{N}$, Kobayashi $\mathrm{H}$, Inukai $\mathrm{M}$, et al. Ligand-functionalizationcontrolled activity of metal-organic framework-encapsulated Pt nanocatalyst toward activation of water. Nano Lett, 2020, 20: 426-432

8 Martínez-Prieto LM, Ferry A, Rakers L, et al. Long-chain NHC-stabilized RuNPs as versatile catalysts for one-pot oxidation/hydrogenation reactions. Chem Commun, 2016, 52: 4768-4771

9 Kabiraz MK, Kim J, Lee WJ, et al. Ligand effect of shape-controlled $\beta$ palladium hydride nanocrystals on liquid-fuel oxidation reactions. Chem Mater, 2019, 31: 5663-5673

10 Levi-Belenkova T, Govorov AO, Markovich G. Orientation-sensitive peptide-induced plasmonic circular dichroism in silver nanocubes. J Phys Chem C, 2016, 120: 12751-12756

11 Han B, Zhu Z, Li Z, et al. Conformation modulated optical activity enhancement in chiral cysteine and Au nanorod assemblies. J Am Chem Soc, 2014, 136: 16104-16107

$12 \mathrm{Li} \mathrm{Y,} \mathrm{Cheng} \mathrm{J,} \mathrm{Li} \mathrm{J,} \mathrm{et} \mathrm{al.} \mathrm{Tunable} \mathrm{chiroptical} \mathrm{properties} \mathrm{from} \mathrm{the}$ plasmonic band to metal-ligand charge transfer band of cysteine-capped molybdenum oxide nanoparticles. Angew Chem Int Ed, 2018, 57: 10236-10240

13 Lee S, Sim K, Moon SY, et al. Controlled assembly of plasmonic nanoparticles: From static to dynamic nanostructures. Adv Mater, 2021, 33: 2007668

14 Giansante C. Enhancing light absorption by colloidal metal chalcogenide quantum dots via chalcogenol(ate) surface ligands. Nanoscale, 2019, 11: 9478-9487

15 Giansante C. Library design of ligands at the surface of colloidal nanocrystals. Acc Chem Res, 2020, 53: 1458-1467

16 Kroupa DM, Vörös M, Brawand NP, et al. Tuning colloidal quantum dot band edge positions through solution-phase surface chemistry modification. Nat Commun, 2017, 8: 15257

17 Huang Z, Zhao ZJ, Zhang Q, et al. A welding phenomenon of dissimilar nanoparticles in dispersion. Nat Commun, 2019, 10: 219

18 Wang Z, He B, Xu G, et al. Transformable masks for colloidal nanosynthesis. Nat Commun, 2018, 9: 563

19 Wang F, Cheng S, Bao Z, et al. Anisotropic overgrowth of metal heterostructures induced by a site-selective silica coating. Angew Chem Int Ed, 2013, 52: 10344-10348

20 Heuer-Jungemann A, Feliu N, Bakaimi I, et al. The role of ligands in the chemical synthesis and applications of inorganic nanoparticles. Chem Rev, 2019, 119: 4819-4880

21 Kim D, Lee DC. Surface ligands as permeation barrier in the growth and assembly of anisotropic semiconductor nanocrystals. J Phys Chem Lett, 2020, 11: 2647-2657

22 Yang TH, Shi Y, Janssen A, et al. Surface capping agents and their roles in shape-controlled synthesis of colloidal metal nanocrystals. Angew
Chem Int Ed, 2020, 59: 15378-15401

23 Zhang Q, Li N, Goebl J, et al. A systematic study of the synthesis of silver nanoplates: Is citrate a "magic" reagent? J Am Chem Soc, 2011, 133: $18931-18939$

24 Sau TK, Murphy CJ. Room temperature, high-yield synthesis of multiple shapes of gold nanoparticles in aqueous solution. J Am Chem Soc, 2004, 126: 8648-8649

25 Koczkur KM, Mourdikoudis S, Polavarapu L, et al. Polyvinylpyrrolidone (PVP) in nanoparticle synthesis. Dalton Trans, 2015, 44: $17883-17905$

26 Xia X, Zeng J, Zhang Q, et al. Recent developments in shape-controlled synthesis of silver nanocrystals. J Phys Chem C, 2012, 116: 21647-21656

27 Wang $\mathrm{Y}, \mathrm{He}$ J, Liu C, et al. Thermodynamics versus kinetics in nanosynthesis. Angew Chem Int Ed, 2015, 54: 2022-2051

28 Feng $\mathrm{Y}$, He J, Wang $\mathrm{H}$, et al. An unconventional role of ligand in continuously tuning of metal-metal interfacial strain. J Am Chem Soc, 2012, 134: 2004-2007

29 He J, Wang Y, Feng Y, et al. Forest of gold nanowires: A new type of nanocrystal growth. ACS Nano, 2013, 7: 2733-2740

30 Wang $\mathrm{Y}, \mathrm{He} \mathrm{J}, \mathrm{Yu} \mathrm{S}$, et al. Effect of thiolated ligands in Au nanowire synthesis. Small, 2017, 13: 1702121

31 Feng Y, Wang Y, He J, et al. Achieving site-specificity in multistep colloidal synthesis. J Am Chem Soc, 2015, 137: 7624-7627

32 Jia J, Liu G, Xu W, et al. Fine-tuning the homometallic interface of Auon-Au nanorods and their photothermal therapy in the NIR-II window. Angew Chem Int Ed, 2020, 59: 14443-14448

33 Tian X, Zong J, Zhou Y, et al. Designing caps for colloidal Au nanoparticles. Chem Sci, 2021, 12: 3644-3650

34 Xiang $\mathrm{T}$, Zong J, $\mathrm{Xu} \mathrm{W}$, et al. Probing the ligand exchange kinetics of phenynyl-based ligands on colloidal Au nanoparticles. Mater Chem Front, 2021, 5: 465-471

35 Huang J, Liu C, Zhu Y, et al. Harnessing structural darkness in the visible and infrared wavelengths for a new source of light. Nat Nanotech, 2015, 11: 60-66

36 Huang J, Zhu Y, Liu C, et al. Unravelling thiol's role in directing asymmetric growth of Au nanorod-Au nanoparticle dimers. Nano Lett, 2016, 16: 617-623

37 Feng J, Xu D, Yang F, et al. Surface engineering and controlled ripening for seed-mediated growth of $\mathrm{Au}$ islands on $\mathrm{Au}$ nanocrystals. Angew Chem Int Ed, 2021, 60: 16958-16964

38 Choueiri RM, Galati E, Thérien-Aubin H, et al. Surface patterning of nanoparticles with polymer patches. Nature, 2016, 538: 79-83

39 Zhou L, Qiu X, Lyu Z, et al. Pd-Au asymmetric nanopyramids: Lateral vs. vertical growth of Au on Pd decahedral seeds. Chem Mater, 2021, 33: $5391-5400$

40 Love JC, Estroff LA, Kriebel JK, et al. Self-assembled monolayers of thiolates on metals as a form of nanotechnology. Chem Rev, 2005, 105: $1103-1170$

41 Wang G, Liu Y, Gao C, et al. Island growth in the seed-mediated overgrowth of monometallic colloidal nanostructures. Chem, 2017, 3: $678-690$

42 Zhou J, Jiang Y, Hou S, et al. Compact plasmonic blackbody for cancer theranosis in the near-infrared II window. ACS Nano, 2018, 12: 26432651

43 Dhiman M, Maity A, Das A, et al. Plasmonic colloidosomes of black gold for solar energy harvesting and hotspots directed catalysis for $\mathrm{CO}_{2}$ to fuel conversion. Chem Sci, 2019, 10: 6594-6603

44 Patterson ML, Weaver MJ. Surface-enhanced Raman spectroscopy as a probe of adsorbate-surface bonding: Simple alkenes and alkynes adsorbed at gold electrodes. J Phys Chem, 1985, 89: 5046-5051

45 Tang $\mathrm{Q}$, Jiang $\mathrm{D}$. Insights into the $\mathrm{PhC} \equiv \mathrm{C} / \mathrm{Au}$ interface. J Phys Chem C, 2015, 119: 10804-10810

46 Chen W, Zuckerman NB, Kang X, et al. Alkyne-protected ruthenium nanoparticles. J Phys Chem C, 2010, 114: 18146-18152

47 Maity $\mathrm{P}$, Tsunoyama $\mathrm{H}$, Yamauchi $\mathrm{M}$, et al. Organogold clusters protected by phenylacetylene. J Am Chem Soc, 2011, 133: 20123-20125

48 Laurentius L, Stoyanov SR, Gusarov S, et al. Diazonium-derived aryl films on gold nanoparticles: evidence for a carbon-gold covalent bond. ACS Nano, 2011, 5: 4219-4227 
49 Sun F, Tang Q. First-principles exploration of the versatile configurations at an alkynyl-protected coinage metal(111) interface. Nanoscale, 2021, 13: 819-831

50 Inkpen MS, Liu ZF, Li H, et al. Non-chemisorbed gold-sulfur binding prevails in self-assembled monolayers. Nat Chem, 2019, 11: 351-358

51 Schlenoff JB, Li M, Ly H. Stability and self-exchange in alkanethiol monolayers. J Am Chem Soc, 1995, 117: 12528-12536

52 Feng $\mathrm{Y}$, Wang $\mathrm{Y}$, Wang $\mathrm{H}$, et al. Engineering "hot" nanoparticles for surface-enhanced Raman scattering by embedding reporter molecules in metal layers. Small, 2012, 8: 246-251

53 Shen W, Lin X, Jiang C, et al. Reliable quantitative SERS analysis facilitated by core-shell nanoparticles with embedded internal standards. Angew Chem, 2015, 127: 7416-7420

54 Feng Y, Wang Y, Song X, et al. Depletion sphere: Explaining the number of Ag islands on Au nanoparticles. Chem Sci, 2017, 8: 430-436

55 Durović MD, Bugarčić ZD, Heinemann FW, et al. Substitution versus redox reactions of gold(III) complexes with L-cysteine, L-methionine and glutathione. Dalton Trans, 2014, 43: 3911-3921

Acknowledgements This work was supported by the National Natural Science Foundation of China (21673117 and 91956109), Jiangsu Provincial Foundation for Specially-Appointed Professor, Jiangsu Science and Technology Plan (BK20211258), Nanjing Tech University (39837102 and 39837140), and SICAM Fellowship from Jiangsu National Synergetic Innovation Center for Advanced Materials.

Author contributions Zong J, Feng Y, and Chen $\mathrm{H}$ conceived the idea and explained the mechanism of structure synthesis, wrote and revised the manuscript. Zong J performed the syntheses and characterization of the nanomaterials. Ren $\mathrm{Q}$, Tian X, and Xiang T participated in the discussion, gave suggestions on the mechanisms, and helped in the artworks.

Conflict of interest The authors declare that they have no conflict of interest.

Supplementary information Supporting data are available in the online version of the paper.

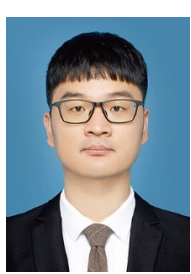

Jianpeng Zong is now a PhD student at the School of Chemistry and Molecular Engineering, Nanjing Tech University, under the supervision of Profs. Yuhua Feng and Hongyu Chen. His research focuses on the synthesis of Janus nanostructures based on interfacial energy control.

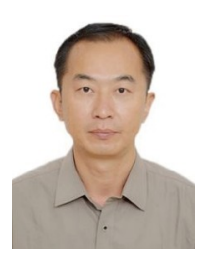

Yuhua Feng received his BSc and MSc degrees from Northeast Normal University and $\mathrm{PhD}$ degree from Nankai University in 2008. He joined Nanjing Tech University in 2017 as professor. His research interest focuses on the design and synthesis of hybrid nanostructures and their applications in plasmonic, SERS and catalysis.

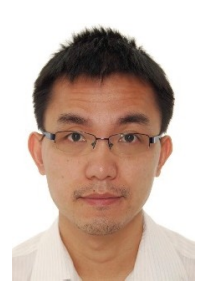

Hongyu Chen received his BSc degree from the University of Science and Technology of China in 1998 and $\mathrm{PhD}$ degree from Yale University in 2004. He joined Nanyang Technological University in 2006 and became associate professor in 2011. He joined Nanjing Tech University as professor in 2016 and now he is a full professor at Westlake University. His research interest focuses on the development of new synthetic control of complex nanostructures.

\section{“补丁”生长模式: 利用炔基配体调控金-金界面}

宗建鹏 ${ }^{1}$, 任权 ${ }^{1}$, 田晓丽 ${ }^{1}$, 项停 ${ }^{1}$, 冯宇华 ${ }^{1^{*}}$, 陈虹宇 ${ }^{1,2,3 *}$

摘要 表面配体在纳米合成中起着关键作用, 扩大适用于纳米合成的 配体范围具有重要意义. 本工作中, 我们系统研究了炔基配体在金种子 上生长金时对金-金界面的调节作用．我们观察到了一种新的“补丁”生 长模式: 具有孔洞以及分支的金补丁“平躺”在金种子表面. 这种生长模 式与传统的弱配体控制的晶面生长以及强配体调控下的非润湿岛状生 长截然不同. 通过控制炔基配体的分子结构及其在金种子表面的排列, 生长的金可以从补丁形状连续调节到岛状，同时其等离子体共振吸收 从可见光区扩展到了近红外光谱区域. 我们相信这类具有中等强度、 能够控制特殊生长模式的新配体的应用, 能够显著提升纳米合成及应 用中的配体控制作用. 